\title{
Progression of hepatic encephalopathy induced by bile duct ligation versus thioacetamide in rats: Regulatory role of apigenin
}

\author{
Ahmed M. Fayez ${ }^{1}$, Dina F. Mansour², Dalia O. Saleh² \\ ${ }^{1}$ Department of Pharmacology, Faculty of Pharmacy, October University for Modern Science and Arts (MSA), Giza, Egypt. \\ ${ }^{2}$ Department of Pharmacology, National Research Centre, Cairo, Egypt.
}

\begin{tabular}{l}
\hline ARTICLE INFO \\
\hline Received on: $16 / 05 / 2021$ \\
Accepted on: $11 / 08 / 2021$ \\
Available online: $05 / 12 / 2021$
\end{tabular}

\section{Key words:}

Hepatic encephalopathy, bile duct ligation, thioacetamide, apigenin, rats.

\begin{abstract}
Hepatic encephalopathy (HE) is the decline in brain functions due to liver insufficiency. A high mortality rate was reported due to the rapid progression of HE from covert to overt, leading to detrimental consequences. This study aims to assess the progression of HE and the potential hepatoprotective and neuroprotective effect of apigenin (APG) in bile duct ligation (BDL) versus thioacetamide (TAA)-induced HE models in rats. Wistar albino rats were divided into eight groups; four groups for the BDL model and the other four groups for the TAA model (100 mg/kg, i.p., thrice weekly for five consecutive weeks). APG (20 mg/kg/day) or lactulose (LAC) ( $8 \mathrm{ml} / \mathrm{kg} / \mathrm{day})$, as the standard, was administered orally for three consecutive weeks starting from day 14 of the experiment. Liver enzymes, total bilirubin, serum ammonia, brain and liver glutathione and malondialdehyde, brain dopamine, hepatic interleukin-6, and nuclear factor kappa B were assessed, as well as the beam walking test and histopathological examinations were carried out. APG showed significant anti-hyperammonemic, anti-oxidant, and anti-inflammatory effects in HE groups. Additionally, improvement in behavioral test and histological image of livers and brains of HE rats treated with APG was observed. In conclusion, APG exerted a significant regulatory role compared to LAC in progression of $\mathrm{HE}$ in BDL and TAA models.
\end{abstract}

\section{INTRODUCTION}

Hepatic encephalopathy (HE) is a neuropsychiatric disorder associated with liver disease after the exclusion of brain disorders (Butterworth, 2016a, 2016b), ranging from personality changes and intellectual impairment to a depressed level of consciousness (Ferenci, 2017). It is considered to be the second most common cause for hospitalization in cirrhotic patients after ascites, thus making $\mathrm{HE}$ an economic burden; although it is easily avoidable, it is the most common cause for readmission (Volk et al., 2012). High mortality rates were reported in patients with high grades of HE in the setting of end stage liver disease prior to coma (Wong et al., 2014).

$\mathrm{HE}$ is categorized to type A due to acute liver failure (ALF), type B due to portosystemic shunting without intrinsic

*Corresponding Author

Dalia O. Saleh, Department of Pharmacology, National Research Centre, Cairo,Egypt.E-mail: doabdelfattah@yahoo.com liver disease (e.g., transjugular intrahepatic portosystemic shunting procedures), and type $\mathrm{C}$ results as a complication of liver cirrhosis (Allampati and Mullen, 2015). Although HE is a reversible syndrome of impaired brain functions accompanying advanced liver failure, it is not a single clinical entity and its diagnosis mainly depends on the exclusion and suspicion with no specific diagnostic test (Ferenci, 2017; Swaminathan et al., 2018). However, liver dysfunction can be treated successfully, HE is associated with poor survival and a high risk of recurrence and changes from covert HE to overt HE (Kaplan and Rossetti, 2011).

The broad spectrum of HE regarding the underlying cause, clinical manifestations, and risk factors making $\mathrm{HE}$ a complex condition and the exact cellular and molecular mechanisms are not yet fully elucidated (Liere et al., 2017). However, the hypotheses of hyperammonemia, neuroinflammation, neurotransmitter system dysfunction and oxidative stress are among the main pathophysiological features of the 
available HE models (Butterworth, 2016c). A wide range of experimental animals have been used in HE research, including large animals, such as dogs, goats, pigs, and rabbits, and rodent, such as rats and mice, using either pharmacological models or surgical models to simulate $\mathrm{HE}$ in acute or chronic liver failure, respectively (Butterworth et al., 2009). Thioacetamide (TAA) is a hepatotoxicant that is extensively used either orally or intraperitoneally to induce ALF in mice and rats (Fontana et al., 1996). The TAA model shows good reproducibility and welldescribed hepatic and cerebral changes as type A HE, producing encephalopathy, metabolic acidosis, high transaminases, abnormal coagulation, and histological centrilobular necrosis (Peeling et al., 1993). Animal models of type C HE lead to decompensated liver cirrhosis and the bile duct ligation (BDL) model induces a reproducible model of biliary cirrhosis in rats, leading to liver failure, portal hypertension, translocation of bacteria, and immune system dysfunction, as well as hyperammonemia and decreased motor activities (Jover et al., 2005). Nevertheless, the BDL model simulates human neuropathology in type $\mathrm{C} \mathrm{HE}$, including Alzheimer type II astrocytosis, altered brain osmolytes, low-grade brain edema, inflammation, and motor activity deficits (Jover et al., 2006).

The addition of rifixamin, lactulose (LAC), 1-ornithine-1aspartate, and ammonia scavengers, such as glycerol phenylbutyrate and ornithine phenylacetate, to $\mathrm{HE}$ treatment guidelines are largely based on good quality clinical trials, although the current research and trials focus on liver support by removing circulating toxins that accumulate in the blood due to liver dysfunction, but more cost-effective studies are required (Hassanein et al., 2011). As HE severely impacts the quality of life of patients due to its unpredictable and complex nature, further treatment options are warranted to prevent the precipitants of $\mathrm{HE}$ and target the pathophysiological hallmarks of the disease, thus imposing actual benefit to HE patients. Therefore, cost-effective and less toxic treatment alternatives are highly needed. Phytochemicals and their derivatives are widely used in liver disease, among which flavonoids have drawn attention due to various pharmacological properties with no significant toxicity (Kumar and Pandey, 2013). Apigenin (APG) is a 4',5,7 trihydroxy flavone that exists in a wide range of plants, and is found at significant levels in many fruits, vegetables, herbs, and spices (Lefort and Blay, 2013). The anti-carcinogenic, anti-metastatic, anti-angiogenic (Shukla and Gupta, 2010), antioxidant properties, hepatoprotective, and antiinflammatory activities of APG have been reported earlier (Ali et al., 2014).

Hence, the current study aims to evaluate the progression of HE in both acute and chronic liver injury using TAA and BDL models, respectively, and the related neurological deficits as a consequence to liver dysfunction along with the identification of the potentials of APG as neuroprotective and hepatoprotective agents in both models of HE.

\section{MATERIAL AND METHODS}

\section{Animals}

Adult male Wistar albino rats weighing 150-250 g were purchased from the Egyptian Company for Production of Vaccines, Sera, and Drugs (EGYVAC; Cairo, Egypt). The animals were housed in plastic cages in the animal house, at the Faculty of Pharmacy, October University for Modern Science and Arts (MSA University). They were kept under appropriate conditions (temperature: $25^{\circ} \mathrm{C} \pm 3^{\circ} \mathrm{C}$; humidity: $50 \%$; and $12 / 12$ hour light/dark cycles) with sufficient food and water. This study was approved by the Research Ethics Committee of Faculty of Pharmacy, October University for Modern Science and Arts (MSA University) (approval number; PH 2/EC2/2018F).

\section{Drugs and chemicals}

APG and TAA were purchased from Amazon (England, UK), whereas LAC was obtained kindly from Abbott (Egypt). The reagent kits, chemicals, and reagents used in the present study were of analytical grade.

\section{BDL rat model}

Common BDL was carried out on Wistar rats to induce cirrhosis type $\mathrm{C} \mathrm{HE}$. The rats were randomly divided into four groups consisting of eight animals in each group (sham surgery, BDL surgery, BDL surgery + APG, and BDL surgery + LAC). APG and LAC were administered after 2 weeks of BDL and daily for three consecutive weeks. Sham and BDL surgery groups received saline intraperitoneally in the same volume, based on the time schedule considered for APG and LAC groups.

The rats were anaesthetized (ketamine $90 \mathrm{mg} / \mathrm{kg}$ and xylazine $12 \mathrm{mg} / \mathrm{kg}$, i.p.). A middle abdominal incision was carried out. Then, after cutting the fascia and muscles, the common bile duct was ligated with a 4-0 silk suture at two points posterior to the hilum of the liver and anterior to the pancreas. The abdominal incision was closed in two layers. In sham animals, the common bile duct was manipulated but not ligated. All animals were maintained for 5 weeks following the surgery (Tag et al., 2015). The principles of laboratory animal care from the Guide for the Care and Use of Laboratory Animals [DHEW publication no. (NIH) 85-23, rev. 985, Office of Science and Health Reports, DRR/NIH, Bethesda, MD, USA] were followed (National Research Council Committee for the Update of the Guide for the C, Use of Laboratory A).

\section{TAA-induced HE rat model}

Type A HE was induced in rats by the administration of TAA at a dose of $100 \mathrm{mg} / \mathrm{kg}$, i.p., thrice weekly for five consecutive weeks (Mansour et al., 2015). To avoid hypoglycemia and electrolyte imbalance, $10 \%$ glucose water mixed with lactate ringer $(25 \mathrm{ml} / \mathrm{kg}$, i.p.) was injected every 12 hours since TAA injections. All rats were caged at $24^{\circ} \mathrm{C}$ with a 12 -hour light/dark cycle and allowed free access to food.

\section{Experimental protocol}

Rats weighing 150-250 g were randomly divided into eight groups; each group consisted of eight rats. The groups were allocated as normal control groups; Sham group and normal saline group, respectively; HE groups; BDL group (Tag et al., 2015), and TAA group, respectively. APG groups received oral APG treatment $(20 \mathrm{mg} / \mathrm{kg} /$ day starting from day 14) for three consecutive weeks in BDL rats and TAA-treated rats (Chen and Zhao, 2016; Sadraei et al., 2017). Finally, LAC groups as standard treatment for HE 
( $8 \mathrm{ml} / \mathrm{kg} /$ day, oral (p.o.), starting from day 14 ) for three consecutive weeks in BDL rats and TAA-treated rats (Jia and Zhang, 2005).

\section{Behavioral test}

Beam walking test is a test showing the capability of the rats to pass through a narrow beam to reach a dark box $(2 \times 100$ $\mathrm{cm})$, which is elevated $1 \mathrm{~m}$ above the floor. To force the rats to pass through the beam, a white light was used at the beginning of the beam. Four trails were made on the day of the test with 5-minute intervals to ensure that the rats were familiar with the beam and knew the existence of the box at the end of the beam. The time needed to cross the beam was recorded and expressed in seconds (Jover et al., 2006).

After 5 weeks, blood was withdrawn from the retroorbital plexus. Then, rats were sacrificed. Liver and brain from each animal were rapidly dissected out, washed, and homogenized using phosphate-buffered saline $(50 \mathrm{mM}$ potassium phosphate, $\mathrm{pH} 7.5$ ) at $4^{\circ} \mathrm{C}$, producing a $20 \%$ homogenate. Liver and brain homogenates were kept at $-80^{\circ} \mathrm{C}$ till time of analyses. Liver and brain tissues were kept in 10\% formalin saline for histopathological examination.

\section{Assessment of liver function}

Activities of serum alanine aminotransferase (ALT) and aspartate aminotransferase (AST) and serum level of alkaline phosphatase (ALP), total bilirubin (TB), and ammonia were determined colorimetrically using commercially available kits (Biodiagnostic, Cairo, Egypt).

\section{Assessment of liver and brain oxidative stress markers}

Malondialdehyde (MDA) was determined using enzymelinked immunosorbent assay (ELISA) kit provided by LifeSpan Biosciences, Inc. (Catalog No. LS-F4236). Reduced glutathione (GSH) was determined using the ELISA kit provided by LifeSpan Biosciences, Inc. (Catalog No. LS-F4612).

\section{Assessment of inflammatory markers}

Interleukin-6 (IL-6) was determined using the ELISA kit provided by LifeSpan Biosciences, Inc. (Catalog No. LSF25921). Nuclear factor kappa-light-chain-enhancers of activated $\mathrm{B}$ cells [nuclear factor kappa B (NF- $\mathrm{BB})$ ] were determined using the ELISA kit provided by LifeSpan Biosciences, Inc. (Catalog No. LS-F21577).

\section{Assessment of dopamine content}

Dopamine was determined using the ELISA kit provided by LifeSpan Biosciences, Inc. (Catalog No. LS-F5364).

\section{Histopathological assessment of rat liver and brain in TAA- and BDL-induced HE}

Autopsy samples were taken from the liver and brain of rats in different groups and fixed in $10 \%$ formalin saline for 24 hours. Washing was carried out in saline and then serial dilutions of alcohol (methyl, ethyl, and absolute ethyl) were used for dehydration. Specimens were cleared in xylene and embedded in paraffin at $56^{\circ}$ in a hot air oven for 24 hours. Paraffin bees wax tissue blocks were prepared for sectioning at 4 microns by slidge microtome. The obtained tissue sections were collected on glass slides, deparaffinized, and stained by hematoxylin and eosin stain, and then examined through the electric light microscope (Bancroft and Gamble, 2008).

\section{Statistical analysis}

All values are presented as means \pm standard error of the means of eight experiments. Comparisons between different groups were carried out using one-way analysis of variance (ANOVA) followed by Tukey-Kramer multiple comparison posthoc test. The difference was considered significant when $p<0.05$. GraphPad prism ${ }^{\circledR}$ software version 6 for Windows (USA) was used to carry out these statistical tests.

\section{RESULTS}

\section{Behavioral assessment}

Effect of APG on beam walking test in BDL versus TTA-induced HE in rats

BDL resulted in a significant increase in time taken by rats to cross the beam as compared to that of sham rats. Treatment with APG (20 ml/kg, p.o.) for three consecutive weeks significantly decreased the time to cross, while treatment with standard LAC ( 8 $\mathrm{ml} / \mathrm{kg}$, p.o.) decreased the time, but it was at a higher time value than APG (Fig. 1).

Administration of TAA (100 mg/kg, i.p.) thrice weekly for five consecutive weeks increased in time to cross the beam when compared to that of normal control rats. Treatment with APG (20 mg/kg, p.o.) for three consecutive weeks significantly decreased the time taken to cross the beam. While treatment with standard LAC ( $8 \mathrm{ml} / \mathrm{kg}$, p.o.) also decreased the time compared to that of normal control (Fig. 1).

\section{Biochemical assessment}

Effect of APG on hepatic function levels and serum ammonia in $B D L$ versus TTA-induced HE in rats

BDL resulted in an increase in AST, ALT, and ALP when compared to that of sham group. Treatment with APG (20 mg/ $\mathrm{kg}$, p.o.) for three consecutive weeks significantly suppressed the elevated AST, ALT, and ALP. Treatment with standard LAC $(8 \mathrm{ml} /$ $\mathrm{kg}$, p.o.) counteracted the elevations in AST, ALT, and ALP, but at a lower value than APG when compared to that of the BDL group (Table 1).

BDL resulted in an increase in TB levels in rats compared to that of sham group. Treatment with APG (20 mg/kg, p.o.) for three consecutive weeks significantly suppressed the elevated TB. Treatment with standard LAC ( $8 \mathrm{ml} / \mathrm{kg}$, p.o.) decreased the levels as well, but at a slight higher value than APG when compared to that of the BDL group (Table 1).

BDL resulted in a significant increase in serum ammonia when compared to that of sham group. Treatment with APG (20 $\mathrm{mg} / \mathrm{kg}$, p.o.) for three consecutive weeks significantly inhibited the elevated ammonia levels. Treatment with standard LAC $(8 \mathrm{ml} /$ $\mathrm{kg}$, p.o.) counteracted the elevations in ammonia levels, and the antihyperammonemic effect of LAC remained significant from that of APG as well as the BDL group (Table 1).

Administration of TAA (100 mg/kg, i.p.) thrice weekly for 5 weeks resulted in an increase in AST, ALT, and ALP levels 


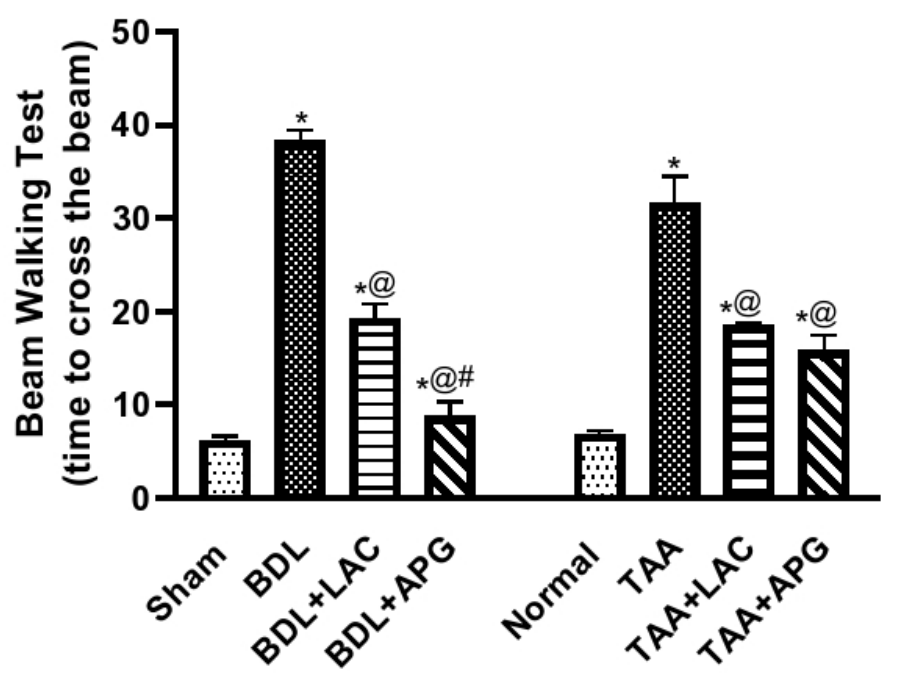

Figure 1. Effect of apigenin on beam walking test in BDL versus TTA induced$\mathrm{HE}$ in rats, respectively. Data is represented as the mean \pm SEM of eight animals. Statistical analysis was completed by one-way ANOVA proceeded by Tukey-Kramer test for multiple comparisons at $p \leq 0.05$. "Significant difference from corresponding control. ${ }^{\circledR}$ Significant difference from HE-induced control. "Significant different from reference drug.

Table 1. Effect of apigenin on serum hepatic function parameters in BDL versus TTA induced-HE in rats.

\begin{tabular}{|c|c|c|c|c|c|c|}
\hline & arameters & $\begin{array}{c}\text { Aspartate } \\
\text { aminotransferase } \\
\text { (AST) } \\
\text { U/I }\end{array}$ & $\begin{array}{c}\text { Alanine aminotransferase } \\
\text { (ALT) } \\
\text { U/l }\end{array}$ & $\begin{array}{c}\text { Alkaline phosphatase } \\
\text { (ALP) } \\
\text { U/I }\end{array}$ & $\underset{(\mathrm{mg} / \mathrm{dL})}{\mathrm{TB}}$ & $\begin{array}{l}\text { Ammonia } \\
(\mathrm{mmol} / \mathrm{ml})\end{array}$ \\
\hline \multirow{8}{*}{ 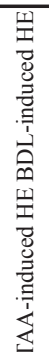 } & Sham control & $9.627 \pm 0.834$ & $100.2 \pm 4.644$ & $113.7 \pm 7.124$ & $0.815 \pm 0.038$ & $52.11 \pm 2.13$ \\
\hline & BDL control & $47.00 \pm 4.177^{\mathrm{a}}$ & $190.2 \pm 6.890^{\mathrm{a}}$ & $199.6 \pm 6.603^{\mathrm{a}}$ & $2.468 \pm 0.0342^{\mathrm{a}}$ & $131.00 \pm 4.48^{\mathrm{a}}$ \\
\hline & $\mathrm{BDL}+\mathrm{LAC}$ & $24.18+1.051^{\mathrm{ab}}$ & $150.5 \pm 7.286^{\mathrm{ab}}$ & $152.1 \pm 5.998^{\mathrm{ab}}$ & $1.037 \pm 0.084^{\mathrm{b}}$ & $63.50 \mathrm{~b} \pm 2.39^{\mathrm{ab}}$ \\
\hline & $\mathrm{BDL}+\mathrm{APG}$ & $16.12 \pm 0.921^{\mathrm{ab}}$ & $100.7 \pm 10.14^{\mathrm{bc}}$ & $114.4 \pm 7.457^{\mathrm{bc}}$ & $1.614 \pm 0.093^{\mathrm{abc}}$ & $95.36 \pm 4.1^{1 \mathrm{abc}}$ \\
\hline & Normal control & $12.37 \pm 1.192$ & $106.2 \pm 9.809$ & $108.5 \pm 8.390$ & $1.068 \pm 0.093$ & $45.14 \pm 2.13$ \\
\hline & TAA & $73.36 \pm 2.209^{\mathrm{a}}$ & $200.1 \pm 10.51^{\mathrm{a}}$ & $215.7 \pm 7.447^{\mathrm{a}}$ & $8.550 \pm 0.489^{a}$ & $134.80 \pm 4.48^{\mathrm{a}}$ \\
\hline & $\mathrm{TAA}+\mathrm{LAC}$ & $50.89+3.725^{\mathrm{ab}}$ & $175.8 \pm 6.478^{\mathrm{ab}}$ & $172.4 \pm 5.546^{\mathrm{ab}}$ & $4.411 \pm 0.206^{\mathrm{ab}}$ & $93.80 \pm 2.39^{\mathrm{ab}}$ \\
\hline & $\mathrm{TAA}+\mathrm{APG}$ & $27.05 \pm 1.843^{\mathrm{abc}}$ & $131.2 \pm 9.502^{\mathrm{abc}}$ & $114.1 \pm 7.896^{\mathrm{bc}}$ & $2.094 \pm 0.102^{\mathrm{abc}}$ & $78.18 \pm 4.18^{\mathrm{abc}}$ \\
\hline
\end{tabular}

Data is represented as the mean \pm SEM of eight animals. Statistical analysis was completed by one-way ANOVA proceeded by Tukey-Kramer test for multiple comparisons at $p \leq 0.05$. aSignificant difference from corresponding control.

${ }^{b}$ Significant difference from HE-induced control.

'Significant different from reference drug.

when compared to that of normal control. Treatment with APG (20 mg/kg, p.o.) for 21 days significantly suppressed the elevated AST, ALT, and ALP. Treatment with standard LAC ( $8 \mathrm{ml} / \mathrm{kg}$, p.o.) decreased the raised AST, ALT, and ALP, but at a lower value than APG when compared to that of the TAA control group (Table 1).

Induction of rats using TAA $(100 \mathrm{mg} / \mathrm{kg}$, i.p.) thrice weekly for three consecutive weeks resulted in an increase in TB levels when compared to that of normal control. Treatment with APG (20 ml/kg, p.o.) for 3 consecutive weeks significantly reversed the elevated TB. Treatment with standard LAC $(8 \mathrm{ml} /$ $\mathrm{kg}$, p.o.) decreased the level of TB, but at a lower value than APG when compared to that of the TAA control group (Table 1).

Administration of TAA (100 mg/kg, i.p.) thrice weekly for 5 weeks resulted in an increase in ammonia levels when compared to that of normal control. Treatment with APG (20 mg/ $\mathrm{kg}$, p.o.) for 3 weeks significantly suppressed the elevated ammonia compared to TAA group as well as standard LAC. Treatment with standard LAC ( $8 \mathrm{ml} / \mathrm{kg}$, p.o.) significantly decreased the raised ammonia levels when compared only to that of the TAA control group (Table 1).

Effect of APG on hepatic IL-6 and NF- $\kappa b$ levels in BDL versus TTA-induced HE in rats

BDL surgery resulted in a significant increase in

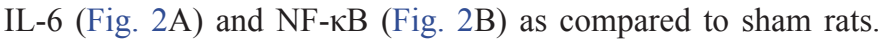
Treatment with APG (20 ml/kg, p.o.) for three consecutive weeks significantly suppressed the elevated IL-6 (Fig. 2A) and NF- $\mathrm{BB}$ (Fig. 2B). While treatment with standard LAC ( $8 \mathrm{ml} / \mathrm{kg}$, p.o.) decreased the levels of IL-6 (Fig. 2A) and NF-אB (Fig. 2B), but at a lower value than APG when compared to that of the BDL control group. 
(A)

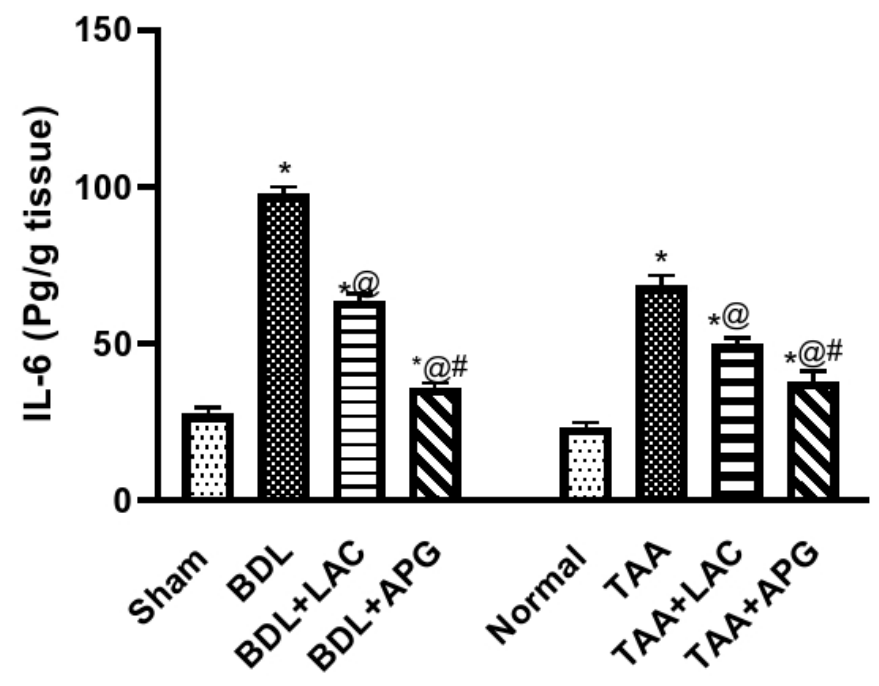

(B)

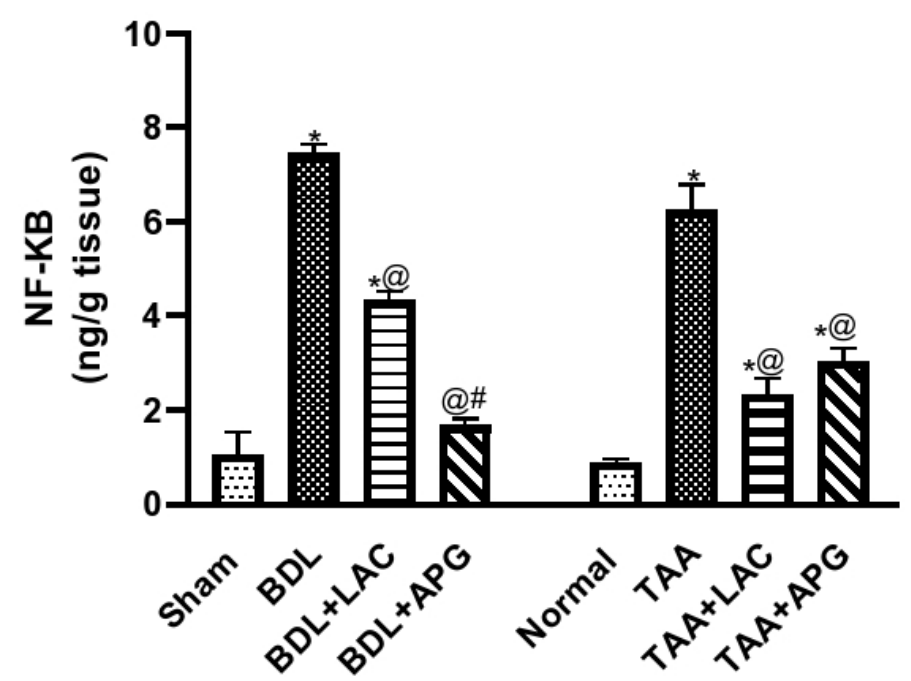

Figure 2. Effect of apigenin on interleukin-6 (A) and NF-kb (B) in BDL versus TTA induced$\mathrm{HE}$ in rats, respectively. Data is represented as the mean \pm SEM of eight animals. Statistical analysis was completed by one-way ANOVA proceeded by Tukey-Kramer test for multiple comparisons at $p \leq 0.05$. "Significant difference from corresponding control. ${ }^{\circledR}$ Significant difference from HE-induced control. "Significant different from reference drug.

Induction of HE using TAA (100 mg/kg, i.p.) thrice weekly for five consecutive weeks resulted in a significant increase in IL-6 (Fig. 2A) and NF- $\mathrm{KB}$ (Fig. 2B) levels when compared to that of normal control. Treatment with APG (20 $\mathrm{ml} / \mathrm{kg} /$ day, p.o.) for three consecutive weeks significantly suppressed the elevations in IL-6 (Fig. 2A) and NF- $\mathrm{KB}$ (Fig. 2B), while treatment with standard LAC $(8 \mathrm{ml} / \mathrm{kg} /$ day, p.o. $)$ decreased the raised levels of IL-6 (Fig. 2A) and NF-KB (Fig. 2B), but at a lower value than APG when compared to that of the TAA control group.

Effect of APG on redox status biomarker in liver and brain tissues in $B D L$ versus TTA-induced HE in rats

BDL resulted in a significant increase in liver and brain MDA while exerting a significant decrease in liver and brain GSH compared to sham rats. Treatment with APG ( $20 \mathrm{ml} / \mathrm{kg}$, p.o.) for three consecutive weeks significantly suppressed the elevated MDA, but further increased the GSH levels. However, treatment with standard LAC ( $8 \mathrm{ml} / \mathrm{kg}$, p.o.) decreased the elevated MDA, but further increased GSH levels in brain and liver tissues at an even lower value than APG as it should, when compared to that of the BDL control group (Table 2).

Induction of HE by TAA (100 mg/kg, i.p.) thrice weekly for five consecutive weeks resulted in an increase in liver and brain MDA and decrease in liver and brain GSH compared to that of normal control. Treatment with APG $(20 \mathrm{ml} / \mathrm{kg}$, p.o) for three consecutive weeks significantly suppressed the elevated MDA, but further increased GSH in liver and brain tissues. However, treatment with standard LAC ( $8 \mathrm{ml} / \mathrm{kg}$, p.o.) decreased MDA, while it increased GSH levels in brain and liver at an even lower 
Table 2. Effect of apigenin on liver and brain oxidative stress biomarkers in BDL versus TTA induced-HE in rats.

\begin{tabular}{|c|c|c|c|c|c|}
\hline & $\frac{\text { Parameters }}{\text { Groups }}$ & $\begin{array}{c}\text { Hepatic MDA } \\
\text { (ng/g tissue) }\end{array}$ & $\begin{array}{c}\text { Hepatic GSH } \\
\text { (ug/g tissue) }\end{array}$ & $\begin{array}{l}\text { Brain MDA } \\
\text { (ng/g tissue) }\end{array}$ & $\begin{array}{c}\text { Brain GSH } \\
\text { (ug/g tissue) }\end{array}$ \\
\hline \multirow{4}{*}{ 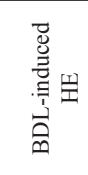 } & Sham Control & $71.03 \pm 5.772$ & $104.09 \pm 2.334$ & $0.6665 \pm 0.052$ & $3.062 \pm 0.1104$ \\
\hline & BDL Control & $188.3 \pm 11.47^{\mathrm{a}}$ & $60.58 \pm 4.733^{\mathrm{a}}$ & $2.538 \pm 0.057^{\mathrm{a}}$ & $1.748 \pm 0.166^{\mathrm{a}}$ \\
\hline & $\mathrm{BDL}+\mathrm{LAC}$ & $142.8+7.200^{\mathrm{ab}}$ & $72.64 \pm 6.261^{\mathrm{ab}}$ & $1.753 \pm 0.157^{\mathrm{ab}}$ & $2.086 \pm 0.091^{b}$ \\
\hline & $\mathrm{BDL}+\mathrm{APG}$ & $106.4 \pm 5.450^{\mathrm{abc}}$ & $91.24 \pm 2.658^{\mathrm{abc}}$ & $0.9694 \pm 0.057^{\mathrm{abc}}$ & $2.891 \pm 0.096^{\mathrm{abc}}$ \\
\hline \multirow{4}{*}{ 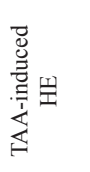 } & Normal control & $42.43 \pm 2.860$ & $24.73 \pm 2.079$ & $0.384 \pm 0.037$ & $2.899 \pm 0.620$ \\
\hline & TAA & $132.2 \pm 8.060^{\mathrm{a}}$ & $7.487 \pm 0.752^{\mathrm{a}}$ & $1.702 \pm 0.073^{\mathrm{a}}$ & $0.620 \pm 0.069^{\mathrm{a}}$ \\
\hline & $\mathrm{TAA}+\mathrm{LAC}$ & $91.90 \pm 2.454^{\mathrm{ab}}$ & $19.66 \pm 1.015^{\mathrm{ab}}$ & $1.246 \pm 0.072^{b}$ & $1.12 \pm 0.060^{\mathrm{b}}$ \\
\hline & $\mathrm{TAA}+\mathrm{APG}$ & $69.45 \pm 3.116^{\mathrm{abc}}$ & $18.71 \pm 1.606^{\mathrm{ab}}$ & $0.867 \pm 0.057^{\mathrm{abc}}$ & $2.195 \pm 0.067^{a b c}$ \\
\hline
\end{tabular}

Data is represented as the mean \pm SEM of eight animals. Statistical analysis was completed by one-way ANOVA proceeded by Tukey-Kramer test for multiple comparisons at $p \leq 0.05$.

aSignificant difference from corresponding control.

${ }^{\mathrm{b} S}$ Significant difference from HE-induced control.

'Significant different from reference drug.

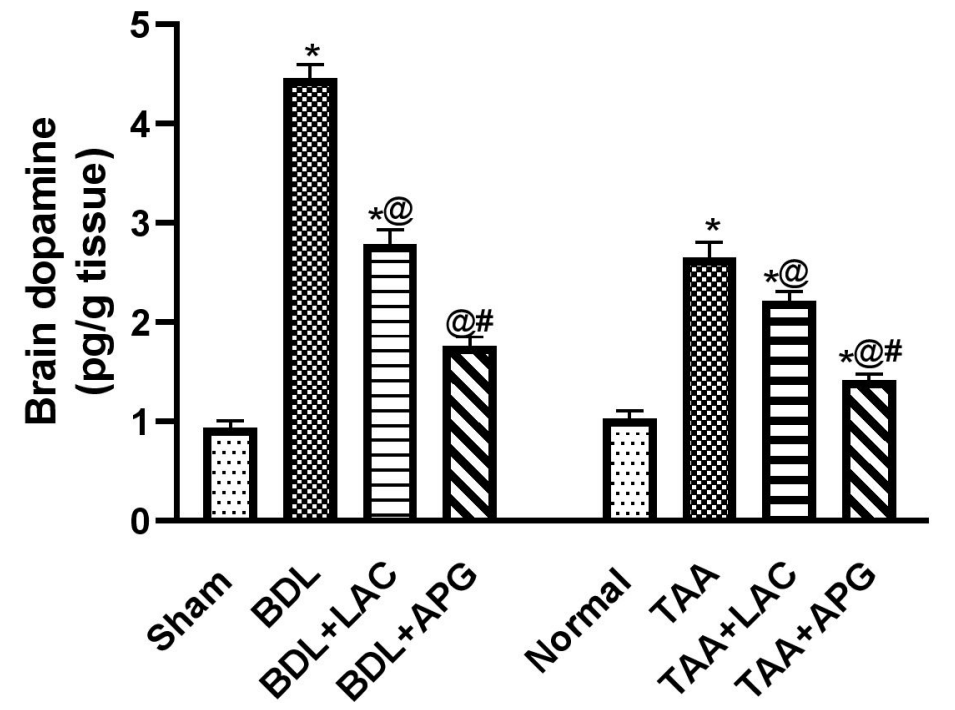

Figure 3. Effect of apigenin on brain dopamine levels in BDL versus TTA induced-HE in rats, respectively. Data is represented as the mean \pm SEM of eight animals. Statistical analysis was completed by one-way ANOVA proceeded by Tukey-Kramer test for multiple comparisons at $p \leq 0.05$. *Significant difference from corresponding control. ${ }^{\circledR}$ Significant difference from HEinduced control. "Significant different from reference drug.

value specifically in the brain tissue for GSH and liver tissue for MDA than APG when compared to that of the TAA control group (Table 2).

Effect of APG on dopamine in brain tissue in BDL versus TTAinduced HE in rats

$\mathrm{BDL}$ in rats resulted in an increase in dopamine when compared to that of sham rats. Treatment with APG $(20 \mathrm{ml} / \mathrm{kg}$, p.o.) for three consecutive weeks significantly suppressed the elevated dopamine. However, treatment with standard LAC ( $8 \mathrm{ml} / \mathrm{kg}$, p.o.) decreased the raised dopamine but at a lower value than APG compared to that of the BDL control group (Fig. 3).

TAA-induced HE (100 mg/kg, i.p.) thrice weekly for five consecutive weeks resulted in an increase in dopamine when compared to that of normal control. Treatment with
APG (20 ml/kg, p.o.) for three consecutive weeks significantly suppressed the elevated dopamine. However, treatment with standard LAC (8 $\mathrm{ml} / \mathrm{kg}$; p.o.) decreased dopamine, but at a higher value than APG compared to that of the TAA control group (Fig. 3).

\section{Histopathological examination}

\section{Effect of APG on liver histopathological alterations in BDL versus TTA-induced HE in rats}

The photomicrographs of the liver of BDL control showed congestion in the central vein, edema, multiple newly formed bile ducts, and fibrosis in association with thickening and fibrosis in the hepatic capsule (Fig. 4IB). The TAAtreated rats showed centrilobular necrosis in the hepatocytes surrounding the central vein associated with fine fibrosis and 
inflammatory cells infiltration with degenerated hepatocytes (Fig. 4IIB).

The liver isolated from BDL rats treated with LAC showed that focal hemorrhage was detected in the parenchyma associated with fine strands of collagen proliferation between the hepatocytes in the parenchyma (Fig. 4IC). On the other hand, liver isolated from TAA-injected rats treated with LAC showed multiple oval cells proliferation was detected surrounding the central vein with degeneration in the hepatocytes (Fig. 4IIC).

After APG treatment to BDL rats, histopathologic pictures showed collagen proliferation with few inflammatory cells infiltration in association with diffuse Kupffer cells proliferation. There was edema and thickening in the capsule but with no hemorrhage (Fig. 4ID). After administration of APG to TAA-injected rats, there were few centrilobular degenerations and sinusoidal dilatations as well as Kupffer cells proliferation and there were few dilatations in the central vein with degeneration in the surrounding adjacent hepatocytes (Fig. 4IID).

\section{Effect of $A P G$ on brain histopathological alterations in $B D L$ versus TTA-induced HE in rats}

Mild congestion was observed in the blood capillaries in the brain tissue isolated from BDL rats; in subiculum, nuclear pyknosis and degeneration were observed. In the cerebellum, it was shown that mild congestion was detected in the meningeal blood vessels (Fig. 5IB). In the striatum, nuclear pyknosis and degeneration were detected in the neurons of the brain tissue isolated from TAA-injected rats associated with intracellular edema (Fig. 5IIB). BDL rats treated with APG have shown no histopathological alteration, in the subiculum also mild congestion in the blood vessels of the meninges covering the cerebellum (Fig. 5ID). Mild intracellular edema was detected in the neurons of rats treated with APG in TAA-injected rats, which is lower than LAC (Fig. 5IID).

\section{DISCUSSION}

The broad range of neuropsychiatric manifestations in $\mathrm{HE}$ reflects complex pathophysiological mechanisms that interrelate to one another. The challenge remains to outline these mechanisms to improve treatment options. In the present study, special attention was paid to type A and type $\mathrm{C} \mathrm{HE}$. As both type $\mathrm{A}-$ and $\mathrm{C}$ HEs are associated with liver insufficiencies either acute or chronic, respectively. However, type B is associated with portalsystemic bypass and no intrinsic hepatocellular disease (Ferenci et al., 1998).

Induction of HE by BDL model in rats showed significant elevations in liver enzyme activities, hyperbilirubinemia, and hyperammonemia in 5 weeks, reflecting haptic injury along with impairment in hepatic excretory functions (Yang et al., 2015). BDL can be used to induce type $\mathrm{C}$ HE in animal models; BDL is a reproducible model in liver damage causing liver failure, jaundice, cirrhosis, portal hypertension, and hyperammonemia; the resultant hyperammonemia is the major etiologic factor in HE (Kwon et al., 2019). BDL rats showed significant decrease in both liver and brain reduced GSH with increased levels of lipid peroxidation biomarker MDA. Liver damage-induced by BDL is due to accumulation of toxic bile acids that trigger oxidative stress and hepatocellular damage, inflammation, and fibrosis (Padillo et al., 2004). The increased oxidative stress also affects the brain antioxidant homeostasis via systemic circulation in adult

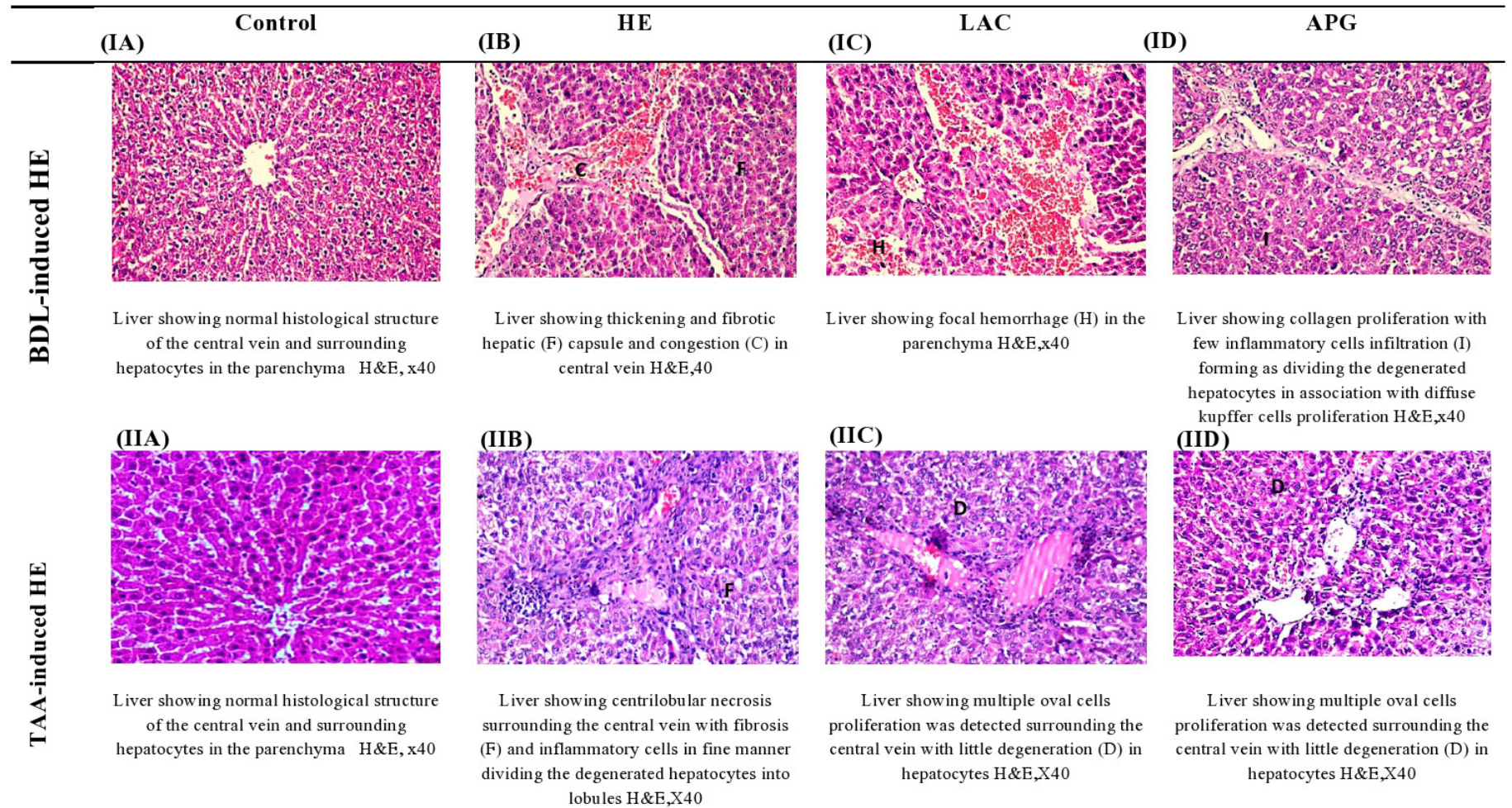

Figure 4. Effect of apigenin on liver histopathological changes in BDL versus TTA induced-HE in rats. 


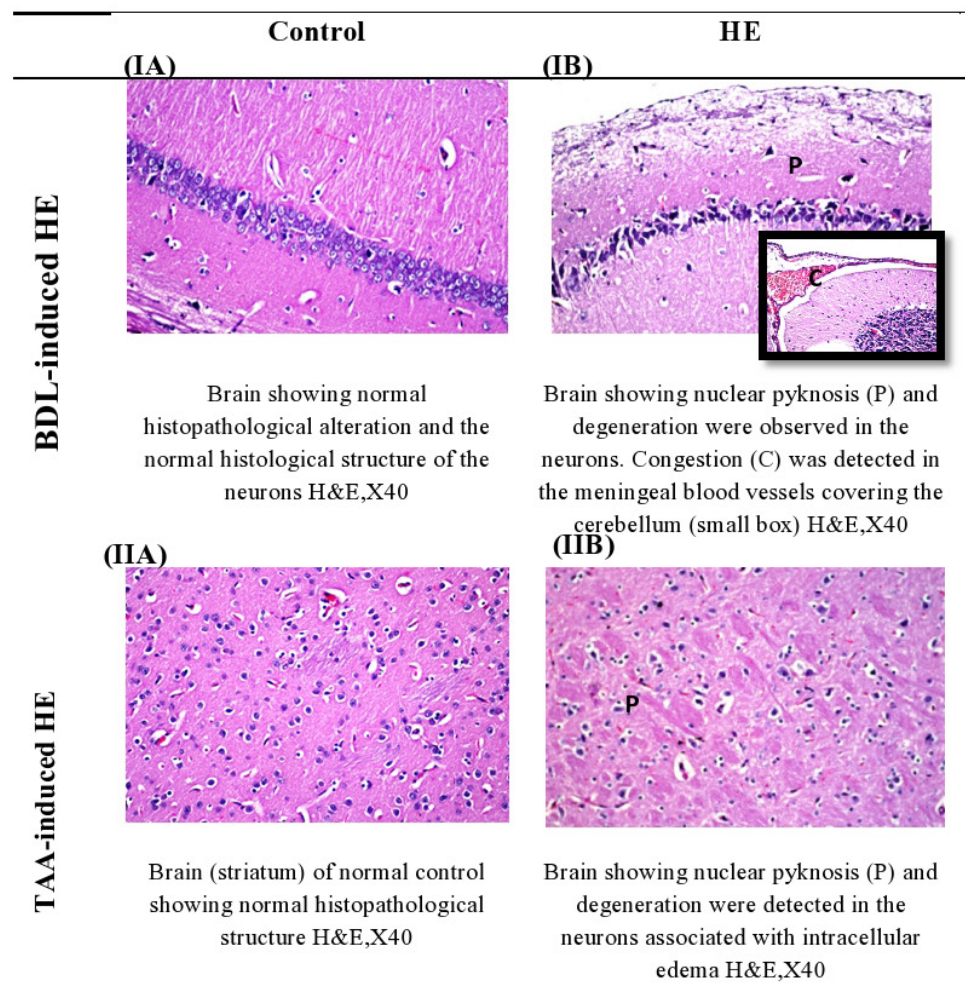

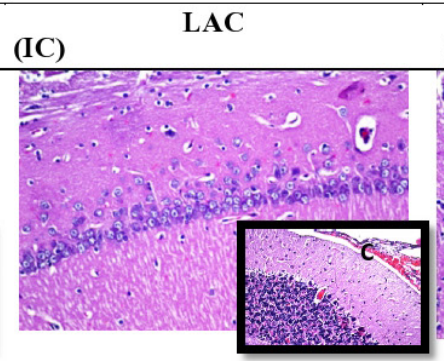

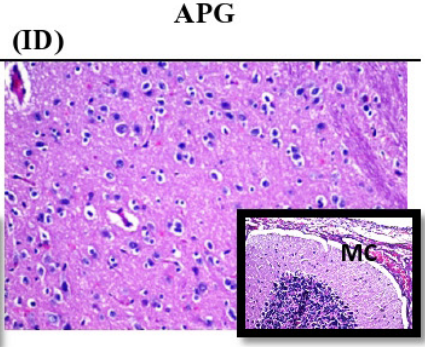

Brain showing normal histopathological alteration. Congestion (C) was detected in the meningeal blood vessels covering the cerebellum (small box) H\&E,X40 (IIC)

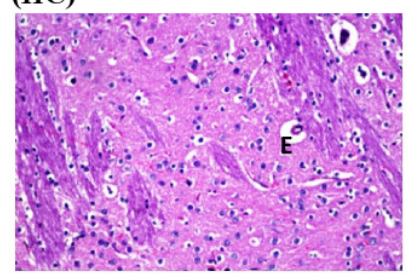

Brain intracellular edema (E) was detected in the cytoplasm of the neurons

$\mathrm{H} \& \mathrm{E}, \mathrm{X} 40$
Brain showing normal histopathological alteration. Mild congestion (MC) in the blood vessels of the meninges covering the cerebellum (small box) H\&E, X40

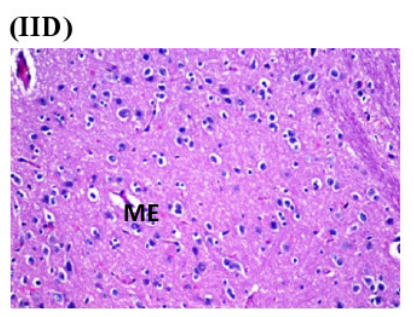

Brain showing mild intracellular edema (ME) H\&E,X40

Figure 5. Effect of apigenin on brain histopathological changes in BDL versus TTA induced-HE in rats.

BDL-induced cholestatic rat (Huang et al., 2009). The altered equilibrium between antioxidant and pro-oxidant activities during cholestasis provoke an inflammatory response (Vazquez-Gil et al., 2004), significant elevations in hepatic NF- $\kappa$ B, and IL-6 in BDL animals was reported in the current study. It is well documented that cholestatic liver injury from biliary obstruction is accentuated by activation of TLR4/NF- $\mathrm{BB}$ signaling pathway and proinflammatory cytokines, such as TNF- $\alpha$ and IL-6, in both human and animal models (Fujiwara et al., 2001; Lechner et al., 1998).

Together, oxidative stress and inflammation during BDL impose significant injury to liver tissues evidenced by accumulation of ammonia and distorted hepatic architecture as supported by the histopathological examinations, such as cholestatic liver congestion of the central vein, multiple newly formed bile ductules, fibrosis, and thickening hepatic capsule. Obstruction of the biliary system causes retention of bile and it has shown that the acute infusion of toxic bile salts is responsible for cholestasis induces hepatocellular necrosis (Woolbright et al., 2015). Chronic bile retention leads to bile duct enlargement and proliferation, it activates hepatic stellate cells to produce collagen, and finally results in periportal and perineoductular fibrosis (Eshraghi et al., 2015).

Unlike cirrhotic patients who can develop slowly from minimal to persistent $\mathrm{HE}$, patients suffering from acute liver insufficiency or ALF progress from altered mental status to coma within days, reporting a high rate of mortality (Misel et al., 2013). In the current study, induction of type A HE by TAA which mimics the ALF along with the neurological dysfunction (Weissenborn et al., 2001) is manifested by elevated levels of ammonia, neuro- inflammation, neurotransmitter system dysfunction, and oxidative stress which in turn leads to behavioral alterations due to $\mathrm{HE}$ confirmed by the histopathological evaluation. Unlike BDL model, TAA causes hepatocellular damage without any cholestasis. This model has been used to clarify changes in the functions of the CNS in HE (Butterworth, 2011). The current data display a significant elevation in liver enzymes, ALT, AST, ALP, bilirubin, and ammonia in TAA-treated rats as reported earlier (Afifi et al., 2020; Mostafa et al., 2017). Furthermore, TAA induced oxidative stress via depleting GSH and elevating MDA in liver and brain tissues of rats, as well as, provoking an inflammatory response evidenced by increased hepatic NF- $\mathrm{BB}$ and IL-6 contents (ElMarasy et al., 2019). Confirming liver injury, the histologic picture of TAA-treated rats showed centrilobular necrosis in the hepatocytes surrounding the central vein associated with fine fibrosis and inflammatory cells infiltration with degenerated hepatocytes. Several studies reported loss of hepatic normal histology with centrilobular necrosis, inflammation, edema, and collagen proliferation and hemorrhage (El-Marasy et al., 2019; Mansour et al., 2015; Mostafa et al., 2017).

The current study has outlined the relationship between hyperammonemia and neurological defects presented by dopamine level, beam walking test, and brain structure abnormalities in both BDL and TAA HE models. As accumulation of glutamine due to hyperammonemia leads to astrocyte swelling (Willard-Mack et al., 1996), the event is observed earlier in cirrhotic patients with minimal HE (Haussinger, 2006) and may contribute to elevated intracranial pressure in patients with fulminant hepatic failure (Blei and Larsen, 1999). Many factors interplay leading to 
morphological abnormalities without diagnostic tool starting from oxidative stress due to hyperammonemia or glutamate leading to astrocyte swelling and excessive dopamine from damaged liver that passes brain blood barrier (BBB) aggravating oxidative stress and astrocyte swelling besides impairment in motor functions (Ding et al., 2014). Our results revealed a marked increase in brain dopamine levels in HE rats of BDL and TAA models, supporting previous data that elevated dopamine is a contributing factor in development of minimal HE in both chronic (Ding et al., 2013) and ALF. Moreover, elevated dopamine levels were accompanied by increase in time of the beam walking test reflecting motor defect in both BDL (Jover et al., 2006; Leke et al., 2012) and TAA-induced HE (Mendez et al., 2009). Morphological changes in brain tissues were confirmed by histopathological examinations revealing neuronal degeneration in cerebellum of BDL animals, while TAA induced evident neuronal degeneration with brain edema as reported earlier (Afifi et al., 2020; El-Marasy et al., 2019; Farjam et al., 2012).

LAC was used as standard treatment for being first-line therapy in management of HE (Pereira et al., 2016). APG is one of the most studied flavonoids, present primarily in significant amount as its glycosylated form in vegetables, fruits, herbs, and plant-based beverages (Hostetler et al., 2017). Recently, various animal models and human clinical trials evidenced the potential neuroprotective effect of APG in depression, Alzheimer's disease, and Parkinson's disease through its antioxidant activity and modulatory effect on $\beta$-amyloid peptide-induced amnesia (Liu et al., 2011; Nabavi et al., 2018). The antioxidant activity of APG was further reported in spinal cord injury (Zhang et al., 2014) along with an anti-inflammatory effect in human AD upon long-term administration of a formulation containing APG (de Font-Reaulx Rojas and Dorazco-Barragan, 2010). APG exerted a hepatoprotective activity in the setting of liver disease via antiinflammatory and antioxidant effects (Saha et al., 2019; Sen and Chakraborty, 2017; Wan and Jiang, 2018). The hepatoprotective and neuroprotective effects of APG in BDL and TAA-induced HE were investigated against LAC in the current study. Data disclosed that administration of APG, at a dose of $20 \mathrm{mg} / \mathrm{kg} /$ day for 3 weeks, exerted significant hepatoprotective and neuroprotective activities compared to LAC at a dose of $8 \mathrm{ml} / \mathrm{kg} /$ day for 3 weeks in BDL and TAA models of HE in rats. APG significantly ameliorated liver enzymes, bilirubin, and ammonia levels when compared to LAC and corresponding HE control groups.

Nevertheless, APG exerted powerful antioxidant and antiinflammatory activity when compared to LAC in both liver and brain of $\mathrm{HE}$ rats via replenishing GSH, modulating MDA levels, and regulating NF- $\mathrm{BB}$ and IL-6, respectively, while improving the histologic picture of both liver and brain in both models of HE, thus confirming its hepatoprotective (Yue et al., 2020) and neuroprotective (Dourado et al., 2020) activities. The potent hepatoprotective activity of APG abrogated hyperammonemia and its neurological consequences in both chronic and ALF as evident by decreased brain oxidative stress and brain dopamine content, while improving motor activity in $\mathrm{HE}$ animals during the beam walking test.

\section{CONCLUSION}

The study concluded that the progression of HE in both chronic and ALF compromise several interrelated factors that contribute to irreversible neurological insults, although diagnosed early, and proper treatments can offend fatal consequences. Hyperammonemia due to liver insufficiency in both BDL and TAA-induced HE could trigger oxidative stress, inflammation, dopamine brain overload, motor impairment, and morphological abnormalities in both models. APG, proven antioxidant, and anti-inflammatory activities exerted hepatoprotective and neuroprotective potentials in type $\mathrm{C}$ and type $\mathrm{A} \mathrm{HE}$ compared to the standard, LAC, in BDL and TAA-induced HE, respectively, in rats. With long-term and cost-effective clinical trials, APG may imply a possible treatment option to $\mathrm{HE}$ in patients with chronic and acute liver disease.

\section{AUTHOR CONTRIBUTIONS}

All authors made substantial contributions to conception and design, acquisition of data, or analysis and interpretation of data; took part in drafting the article or revising it critically for important intellectual content; agreed to submit to the current journal; gave final approval of the version to be published; and agree to be accountable for all aspects of the work. All the authors are eligible to be an author as per the international committee of medical journal editors (ICMJE) requirements/guidelines.

\section{FUNDING}

There is no funding to report.

\section{CONFLICTS OF INTEREST}

The authors report no financial or any other conflicts of interest in this work.

\section{PUBLISHER'S NOTE}

This journal remains neutral with regard to jurisdictional claims in published institutional affiliation.

\section{REFERENCES}

Afifi NA, Ramadan A, Erian EY, Sedik AA, Amin MM, Hassan A, Saleh DO. Synergistic effect of aminoguanidine and L-carnosine against thioacetamide-induced hepatic encephalopathy in rats: behavioral, biochemical and ultra structural evidences. Can J Physiol Pharmacol, 2020; 99:332-47.

Ali F, Rahul, Naz F, Jyoti S, Siddique YH. Protective effect of apigenin against $\mathrm{N}$-nitrosodiethylamine (NDEA)-induced hepatotoxicity in albino rats. Mutat Res Genet Toxicol Environ Mutagen, 2014 767:13-20.

Allampati S, Mullen KD. Nomenclature and definition of hepatic encephalopathy — an update. Clin Liver Dis (Hoboken), 2015; 5(3):68-70.

Bancroft JD, Gamble M. Theory and practice of histological techniques. Elsevier Health Sciences, London, UK, 2008.

Blei AT, Larsen FS. Pathophysiology of cerebral edema in fulminant hepatic failure. J Hepatol, 1999; 31(4):771-6.

Butterworth RF, Norenberg MD, Felipo V, Ferenci P, Albrecht J, Blei AT, Members of the ISHEN Commission on Experimental Models of HE. Experimental models of hepatic encephalopathy: ISHEN guidelines. Liver Int, 2009; 29(6):783-8.

Butterworth RF. Hepatic encephalopathy: a central neuroinflammatory disorder? Hepatology, 2011; 53(4):1372-6.

Butterworth RF. Neurosteroids in hepatic encephalopathy: novel insights and new therapeutic opportunities. J Steroid Biochem Mol Biol, 2016a; 160:94-7.

Butterworth RF. Pathogenesis of hepatic encephalopathy in cirrhosis: the concept of synergism revisited. Metab Brain Dis, 2016b; 31(6):1211-5. 
Butterworth RF. The concept of the inflamed brain in acute liver failure: mechanisms and new therapeutic opportunities. Metab Brain Dis, 2016c; 31(6):1283-7.

Chen L, Zhao W. Apigenin protects against bleomycin-induced lung fibrosis in rats. Exp Ther Med, 2016; 11(1):230-4.

de Font-Reaulx Rojas E, Dorazco-Barragan G. Clinical stabilisation in neurodegenerative diseases: clinical study in phase II. Rev Neurol, 2010; 50(9):520-8.

Ding S, Liu L, Jing H, Xie J, Wang X, Mao J, Chen B, Zhuge Q. Dopamine from cirrhotic liver contributes to the impaired learning and memory ability of hippocampus in minimal hepatic encephalopathy. Hepatol Int, 2013; 7(3):923-36.

Ding S, Yang J, Liu L, Ye Y, Wang X, Hu J, Chen B, Zhuge Q. Elevated dopamine induces minimal hepatic encephalopathy by activation of astrocytic NADPH oxidase and astrocytic protein tyrosine nitration. Int J Biochem Cell Biol, 2014; 55:252-63.

Dourado NS, Souza CDS, de Almeida MMA, Bispo da Silva A, Dos Santos BL, Silva VDA, De Assis AM, da Silva JS, Souza DO, Costa MD, Butt AM. Neuroimmunomodulatory and neuroprotective effects of the flavonoid apigenin in in vitro models of neuroinflammation associated with Alzheimer's disease. Front Aging Neurosci, 2020; 12:119.

El-Marasy SA, El Awdan SA, Abd-Elsalam RM. Protective role of chrysin on thioacetamide-induced hepatic encephalopathy in rats. Chem Biol Interact, 2019; 299:111-9.

Eshraghi T, Eidi A, Mortazavi P, Asghari A, Tavangar SM. Magnesium protects against bile duct ligation-induced liver injury in male Wistar rats. Magnesium Res, 2015; 28(1):32-45.

Farjam M, Dehdab P, Abbassnia F, Mehrabani D, Tanideh $\mathrm{N}$, Pakbaz S, Imanieh MH. Thioacetamide-induced acute hepatic encephalopathy in rat: behavioral, biochemical and histological changes. Iran Red Crescent Med J, 2012; 14(3):164-70. 5(2):138-47.

Ferenci P. Hepatic encephalopathy. Gastroenterol Rep, 2017;

Ferenci P, Lockwood A, Mullen K, Tarter R, Weissenborn K, Blei AT. Hepatic encephalopathy - definition, nomenclature, diagnosis, and quantification: final report of the working party at the 11th World Congresses of Gastroenterology, Vienna, 1998. Hepatology, 2002; 35(3):716-21.

Fontana L, Moreira E, Torres MI, Fernandez MI, Rios A, Sanchez de Medina F, Gil A. Serum amino acid changes in rats with thioacetamideinduced liver cirrhosis. Toxicology, 1996; 106(1-3):197-206.

Fujiwara Y, Shimada M, Yamashita Y, Adachi E, Shirabe K, Takenaka K, Sugimachi K. Cytokine characteristics of jaundice in mouse liver. Cytokine, 2001; 13(3):188-91.

Hassanein TI, Schade RR, Hepburn IS. Acute-on-chronic liver failure: extracorporeal liver assist devices. Curr Opin Crit Care, 2011; 17(2):195-203.

Haussinger D. Low grade cerebral edema and the pathogenesis of hepatic encephalopathy in cirrhosis. Hepatology, 2006; 43(6):1187-90.

Huang LT, Tiao MM, Tain YL, Chen CC, Hsieh CS. Melatonin ameliorates bile duct ligation-induced systemic oxidative stress and spatial memory deficits in developing rats. Pediatr Res, 2009; 65(2):176-80.

Hostetler GL, Ralston RA, Schwartz SJ. Flavones: food sources, bioavailability, metabolism, and bioactivity. Adv Nutr, 2017; $8(3): 423-35$.

Jia L, Zhang MH. Comparison of probiotics and lactulose in the treatment of minimal hepatic encephalopathy in rats. World J Gastroenterol, 2005; 11(6):908-11.

Jover R, Madaria E, Felipo V, Rodrigo R, Candela A, Compan A. Animal models in the study of episodic hepatic encephalopathy in cirrhosis. Metab Brain Dis, 2005; 20(4):399-408.

Jover R, Rodrigo R, Felipo V, Insausti R, Saez-Valero J, GarciaAyllon MS, Suarez I, Candela A, Compan A, Esteban A, Cauli O. Brain edema and inflammatory activation in bile duct ligated rats with dietinduced hyperammonemia: a model of hepatic encephalopathy in cirrhosis. Hepatology, 2006; 43(6):1257-66.
Kaplan PW, Rossetti AO. EEG patterns and imaging correlations in encephalopathy: encephalopathy part II. J Clin Neurophysiol, 2011; 28(3):233-51.

Kumar S, Pandey AK. Chemistry and biological activities of flavonoids: an overview. Sci World J, 2013; 2013:162750.

Kwon KW, Nam Y, Choi WS, Kim TW, Kim GM, Sohn UD. Hepatoprotective effect of sodium hydrosulfide on hepatic encephalopathy in rats. Korean J Physiol Pharmacol, 2019; 23(4):263-70.

Lechner AJ, Velasquez A, Knudsen KR, Johanns CA, Tracy Jr TF, Matuschak GM. Cholestatic liver injury increases circulating TNF- $\alpha$ and IL-6 and mortality after Escherichia coli endotoxemia. Am J Respir Crit Care Med, 1998; 157(5):1550-8.

Lefort EC, Blay J. Apigenin and its impact on gastrointestinal cancers. Mol Nutr Food Res, 2013; 57(1):126-44.

Leke R, de Oliveira DL, Mussulini BH, Pereira MS, Kazlauckas V, Mazzini G, Hartmann CR, Silveira TR, Simonsen M, Bak LK, Waagepetersen HS. Impairment of the organization of locomotor and exploratory behaviors in bile duct-ligated rats. PLoS One, 2012; 7(5):e36322.

Liere V, Sandhu G, DeMorrow S. Recent advances in hepatic encephalopathy. F1000Res, 2017; 6:1637

Liu R, Zhang T, Yang H, Lan X, Ying J, Du G. The flavonoid apigenin protects brain neurovascular coupling against amyloid-beta(2)(5) (-)(3)(5)-induced toxicity in mice. J Alzheimers Dis, 2011; 24(1):85-100.

Mansour DF, Nada SA, Eldenshary EES, Elmahmoudy BM, AbdElgayed SS. Antioxidant and hypo-ammonemic activities of alphalactalbumin and vitamin $\mathrm{C}$ in thioacetamide-induced liver and brain damage in rats. J Appl Pharm Sci, 2015; 5:072-81.

Mendez M, Mendez-Lopez M, Lopez L, Aller MA, Arias J, Arias JL. Associative learning deficit in two experimental models of hepatic encephalopathy. Behav Brain Res, 2009; 198(2):346-51.

Misel ML, Gish RG, Patton H, Mendler M. Sodium benzoate for treatment of hepatic encephalopathy. Gastroenterol Hepatol (NY), 2013; 9(4):219-27.

Mostafa RE, Salama AAA, Abdel-Rahman RF, Ogaly HA. Hepato- and neuro-protective influences of biopropolis on thioacetamideinduced acute hepatic encephalopathy in rats. Can J Physiol Pharmacol, 2017; 95(5):539-47.

Nabavi SF, Khan H, D'onofrio G, Samec D, Shirooie S, Dehpour AR, Arguelles S, Habtemariam S, Sobarzo-Sanchez E. Apigenin as neuroprotective agent: of mice and men. Pharmacol Res, 2018; 128:359-65.

National Research Council Committee for the Update of the Guide for the C, Use of Laboratory A. The National Academies Collection: reports funded by National Institutes of Health. Guide for the care and use of laboratory animals. National Academies Press, Washington, DC, 2011.

Padillo FJ, Cruz A, Navarrete C, Bujalance I, Briceno J, Gallardo JI, Marchal T, Caballero R, Tunez I, Muntane J, Montilla P. Melatonin prevents oxidative stress and hepatocyte cell death induced by experimental cholestasis. Free Radic Res, 2004; 38(7):697-704.

Peeling J, Shoemaker L, Gauthier T, Benarroch A, Sutherland GR, Minuk GY. Cerebral metabolic and histological effects of thioacetamideinduced liver failure. Am J Physiol, 1993; 265(3 Pt 1):G572-8.

Pereira K, Carrion AF, Salsamendi J, Doshi M, Baker R, Kably I. Endovascular management of refractory hepatic encephalopathy complication of transjugular intrahepatic portosystemic shunt (TIPS): comprehensive review and clinical practice algorithm. Cardiovasc Intervent Radiol, 2016; 39(2):170-82.

Sadraei H, Asghari G, Khanabadi M, Minaiyan M. Antiinflammatory effect of apigenin and hydroalcoholic extract of Dracocephalum kotschyi on acetic acid-induced colitis in rats. Res Pharm Sci, 2017; 12(4):322-9.

Saha P, Talukdar AD, Nath R, Sarker SD, Nahar L, Sahu J, Choudhury MD. Role of natural phenolics in hepatoprotection: a mechanistic review and analysis of regulatory network of associated genes. Front Pharmacol, 2019; 10:509. 
Shukla S, Gupta S. Apigenin: a promising molecule for cancer prevention. Pharm Res, 2010; 27(6):962-78.

Sen S, Chakraborty R. Herbs, gastrointestinal protection, and oxidative stress. Gastrointestinal tissue. Elsevier, pp 259-74, 2017.

Swaminathan M, Ellul MA, Cross TJ. Hepatic encephalopathy: current challenges and future prospects. Hepat Med, 2018; 10:1-11.

Tag CG, Sauer-Lehnen S, Weiskirchen S, Borkham-Kamphorst $\mathrm{E}$, Tolba RH, Tacke F, Weiskirchen R. Bile duct ligation in mice: induction of inflammatory liver injury and fibrosis by obstructive cholestasis. J Vis Exp, 2015; 10(96):52438.

Vazquez-Gil MJ, Mesonero MJ, Flores $\mathrm{O}$, Criado $\mathrm{M}$, Hidalgo F, Arevalo MA, Sanchez-Rodriguez A, Tunon MJ, LopezNovoa JM, Esteller A. Sequential changes in redox status and nitric oxide synthases expression in the liver after bile duct ligation. Life Sci, 2004; 75(6):717-32.

Volk ML, Tocco RS, Bazick J, Rakoski MO, Lok AS. Hospital readmissions among patients with decompensated cirrhosis. Am J Gastroenterol, 2012; 107(2):247--52.

Wan L, Jiang JG. Protective effects of plant-derived flavonoids on hepatic injury. J Funct Foods, 2018; 44:283-91.

Weissenborn K, Ennen JC, Schomerus H, Ruckert N, Hecker H. Neuropsychological characterization of hepatic encephalopathy. J Hepatol, 2001; 34(5):768-73.

Willard-Mack CL, Koehler RC, Hirata T, Cork LC, Takahashi $\mathrm{H}$, Traystman RJ, Brusilow SW. Inhibition of glutamine synthetase reduces ammonia-induced astrocyte swelling in rat. Neuroscience, 1996; 71(2):589-99.
Woolbright BL, Dorko K, Antoine DJ, Clarke JI, Gholami P, Li F, Kumer SC, Schmitt TM, Forster J, Fan F, Jenkins RE, Park BK, Hagenbuch B, Olyaee M, Jaeschke H. Bile acid-induced necrosis in primary human hepatocytes and in patients with obstructive cholestasis. Toxicol Appl Pharmacol, 2015; 283(3):168-77.

Wong RJ, Gish RG, Ahmed A. Hepatic encephalopathy is associated with significantly increased mortality among patients awaiting liver transplantation. Liver Transpl, 2014; 20(12):1454-61.

Yang Y, Chen B, Chen Y, Zu B, Yi B, Lu K. A comparison of two common bile duct ligation methods to establish hepatopulmonary syndrome animal models. Lab Anim, 2015; 49(1):71-9.

Yue S, Xue N, Li H, Huang B, Chen Z, Wang X. Hepatoprotective effect of apigenin against liver injury via the non-canonical NF-kappaB pathway in vivo and in vitro. Inflammation, 2020; 43(5):1634-48.

Zhang F, Li F, Chen G. Neuroprotective effect of apigenin in rats after contusive spinal cord injury. Neurol Sci, 2014; 35(4):583-8.

How to cite this article:

Fayez AM, Mansour DF, Saleh DO. Progression of hepatic encephalopathy induced by bile duct ligation versus thioacetamide in rats: Regulatory role of apigenin. J Appl Pharm Sci, 2021; 11(12):158-168. 